\title{
Some results on quasipolyhedral convexity
}

\section{M.D. Fajardo and M.A. López}

Departamento de Estadistica e Investigación Operativa Facultad de Ciencias, Universidad de Alicante, Spain

E-mail:md.fajardo@ua.esmarco.antonio@ua.es

\begin{abstract}
The objective of this paper is to analyse under what well-known operations the class of quasipolyhedral convex functions, which can be regarded as an extension of that of polyhedral convex functions, is closed. The operations that will be considered are those that preserve polyhedral convexity, such that the image and the inverse image under linear transformations, right scalar multiplication (including the case where $\lambda=0^{+}$) and pointwise addition.
\end{abstract}

Key Words: Semi-infinite linear inequality systems, polyhedral and quasipolyhedral convex sets, reccesion function.

AMS subject classification: 90C34, 52A41, 90C25, 52A20.

\section{Introduction}

A polyhedral convex set in $\mathbb{R}^{n}$ is a non-empty set that can be expressed as the intersection of some finite collection of closed half-spaces; i.e., as the set of solutions of some system of inequalities of the form

$$
\left\{\left\langle a_{i}, x\right\rangle \leq b_{i}, i=1,2, \ldots, m\right\},
$$

with $x$ and $a_{i}$ in $\mathbb{R}^{n}, b_{i}$ in $\mathbb{R}$, for $i=1,2, \ldots, m$, and $\langle.,$.$\rangle representing the usual$ inner product in $\mathbb{R}^{n}$.

Bounded polyhedral convex sets are called polytopes, and a polyhedral convex function $f$ on $\mathbb{R}^{n}$ is a convex one whose epigraph,

$$
\text { epi } f:=\left\{\left(\begin{array}{l}
x \\
\alpha
\end{array}\right) \in \mathbb{R}^{n+1} \mid f(x) \leq \alpha \text { and } x \in \operatorname{dom} f\right\} \text {, }
$$

is a polyhedral convex set in $\mathbb{R}^{n+1}$, where $\operatorname{dom} f:=\left\{x \in \mathbb{R}^{n} \mid f(x)<+\infty\right\}$ is the effective domain of $f$.

The property of being polyhedral is preserved under many operations. In Rockafellar (1970), Theorem 19.3, it is shown that the image (respectively, the inverse image) of a polyhedral convex set in $\mathbb{R}^{n}$ (respectively, in $\mathbb{R}^{m}$ ) under a linear transformation $A$ from $\mathbb{R}^{n}$ to $\mathbb{R}^{m}$, is a polyhedral convex set in $\mathbb{R}^{m}$ (respectively, in $\mathbb{R}^{n}$ ). As a consequence of Corollary 19.3.1 in Rockafellar (1970), for each polyhedral convex function $f$ on $\mathbb{R}^{n}$ (respectively, 
$g$ on $\mathbb{R}^{m}$ ), the convex function

$$
(A f)(y):=\inf \{f(x) \mid A x=y\}
$$

on $\mathbb{R}^{m}$ (respectively, the convex function $(g A)(x):=g(A x)$ on $\mathbb{R}^{n}$ ) is polyhedral too.

Moreover, if $f_{1}$ and $f_{2}$ are proper convex polyhedral functions on $\mathbb{R}^{n}$, then $f_{1}+f_{2}$ is polyhedral (Theorem 19.4 in Rockafellar (1970)).

It is also shown in Rockafellar (1970), Theorem 19.5, that for every nonempty polyhedral convex set $C$ in $\mathbb{R}^{n}$, the convex set

$$
\lambda C:=\{\lambda x \mid x \in C\}
$$

is polyhedral, for every $\lambda \in \mathbb{R}$, and its recession cone

$$
0^{+} C:=\left\{v \in \mathbb{R}^{n} \mid x+\alpha v \in C, \text { for every } x \in C \text { and every } \alpha \geq 0\right\}
$$

is polyhedral. From these results, it can be derived (Corollary 19.5.1 in Rockafellar (1970)) that given a proper polyhedral convex function $f$ on $\mathbb{R}^{n}$, the convex function

$$
(f \lambda)(x):=\lambda f\left(\lambda^{-1} x\right),
$$

for $\lambda>0$, is polyhedral, as well as its recession function $f 0^{+}$, defined as the convex function whose epigraph is $0^{+}$(epi $f$ ).

The concept of polyhedral convex set has been generalized in the following way: we say that a non-empty convex set is quasipolyhedral if its intersections with polytopes are polytopes. Hence, a convex function on $\mathbb{R}^{n}$ is said to be quasipolyhedral if its epigraph is a quasipolyhedral convex set in $\mathbb{R}^{n+1}$.

Our objective is to investigate under which operations the class of quasipolyhedral functions is closed.

In order to simplify the proofs, we shall suppose that $f$ is a finite-valued convex function; i.e., $\operatorname{dom} f=\mathbb{R}^{n}$. Then $f$ is continuous on $\mathbb{R}^{n}$ and, therefore, closed.

For any finite-valued convex function $f$ on $\mathbb{R}^{n}$, if epi $f \subset \mathbb{R}^{n+1}$ is the solution set of the system

$$
\sigma=\left\{\left\langle a_{t}, x\right\rangle-x_{n+1} \leq b_{t}, t \in T\right\},
$$

with $x$ and $a_{t}$ in $\mathbb{R}^{n}, b_{t}$ in $\mathbb{R}$ and $T$ being an arbitrary index set, we shall say that $\sigma$ is a representation of $f$ by means of affine minorants associated with the inequalities of $\sigma$. Moreover, $\sigma$ in (1.1) is a representation of $f$ if and only if $f$ can be expressed as

$$
f(x)=\sup \left\{\left\langle a_{t}, x\right\rangle-b_{t}, t \in T\right\} .
$$


Let $C$ be a non-empty set in $\mathbb{R}^{n}$ and $\sigma$ a representation of a finite-valued convex function $f$. We shall use the notation

$$
T(C):=\bigcup\{T(x), x \in C\},
$$

where $T(x)$ is a the set of sup-active indices at $x$; i.e.,

$$
T(x):=\left\{t \in T \mid f(x)=\left\langle a_{t}, x\right\rangle-b_{t}\right\} .
$$

Finite-valued quasipolyhedral convex functions can be characterized in the following way (Proposition 4 in Fajardo et al. (2006)):

Proposition 1.1 A finite-valued convex function $f$ is quasipolyhedral if and only if there exists a representation of $f, \sigma=\left\{\left\langle a_{t}, x\right\rangle-x_{n+1} \leq b_{t}, t \in T\right\}$, such that $T(C)$ is a non-empty finite index subset, for every non-empty compact set $C$ in $\mathbb{R}^{n}$.

From now on we denote by $\mathcal{Q}^{k}$ the set of finite-valued quasipolyhedral convex functions on $\mathbb{R}^{k}$, for every $k \in \mathbb{N}$.

Some additional notation will be used. The convex (conical, linear) hull of a non-empty set $X$ is denoted by $\operatorname{conv} X$ (cone $X, \operatorname{span} X$, respectively) and, from the topological side, $\operatorname{cl} X$ represents the closure of $X$. Finally, the Euclidean norm is represented by $\|$.$\| .$

\section{Applying some operators on $\mathcal{Q}^{k}$}

Proposition 2.1 Let A be a linear transformation from $\mathbb{R}^{n}$ to $\mathbb{R}^{m}$, with $m \geq n$. Then the convex set in $\mathbb{R}^{m}$

$$
A C:=\{A x \mid x \in C\}
$$

is quasipolyhedral, for every quasipolyhedral convex set $C$ in $\mathbb{R}^{n}$, if and only if either $A x=0_{m}$, for all $x \in \mathbb{R}^{n}$, or $\operatorname{ker} A=\left\{0_{n}\right\}$. If the latter condition holds, the convex set in $\mathbb{R}^{n}$

$$
A^{-1} D:=\left\{x \in \mathbb{R}^{n} \mid A x \in D\right\},
$$

provided that it is non-empty, is quasipolyhedral, for every quasipolyhedral convex set $D$ in $\mathbb{R}^{m}$.

Proof. If $A x=0_{m}$, for all $x \in \mathbb{R}^{n}, A C$ is a singleton, which trivially is a quasipolyhedral convex set. Thus, let us assume that $\operatorname{ker} A=\left\{0_{n}\right\}$, in which case $A$ is a one-to-one mapping between $\mathbb{R}^{n}$ and $A \mathbb{R}^{n}$. 
Let $P$ be a polytope in $\mathbb{R}^{m}$, such that $(A C) \cap P \neq \emptyset$. We shall prove that $(A C) \cap P$ is a polytope. Since it is bounded, it is enough to prove that it is a polyhedral convex set in $\mathbb{R}^{m}$.

We have $A^{-1}((A C) \cap P)=C \cap\left(A^{-1} P\right) \neq \emptyset$. By Theorem 19.3 in Rockafellar (1970), $A^{-1} P$ is a polyhedral convex set in $\mathbb{R}^{n}$, and Corollary 8.3.4 in Rockafellar (1970), together with the assumption ker $A=\left\{0_{n}\right\}$, yields

$$
0^{+}\left(A^{-1} P\right)=A^{-1}\left(0^{+} P\right)=A^{-1}\left(0_{m}\right)=0_{n} .
$$

So, $A^{-1} P$ is a polytope, as well as $C \cap\left(A^{-1} P\right)$. Then, applying Theorem 19.3 in Rockafellar (1970) again, the set

$$
\begin{aligned}
A\left(C \cap\left(A^{-1} P\right)\right) & =(A C) \cap A\left(A^{-1} P\right) \\
& =(A C) \cap A\left(A^{-1}\left(P \cap A \mathbb{R}^{n}\right)\right) \\
& =(A C) \cap\left(P \cap A \mathbb{R}^{n}\right) \\
& =(A C) \cap P
\end{aligned}
$$

is a polyhedral convex set in $\mathbb{R}^{m}$.

Next we proceed with the proof of the converse statement. So, we assume that $A C$ is quasipolyhedral, for every quasipolyhedral convex set $C$.

If $A x \neq 0_{m}$, for some $x \in \mathbb{R}^{n}$, and ker $A \neq\left\{0_{n}\right\}$, we can choose two vectors $u \in \operatorname{ker} A$ and $v \in \mathbb{R}^{n} \backslash \operatorname{ker} A$, such that $\|u\|=\|v\|=1$. Consider the countable set

$$
S:=\left\{s^{k}=k u+\frac{1}{k} v, k \in \mathbb{N}\right\}
$$

and define $C:=\operatorname{conv} S+$ cone $\{u\}$. In $L:=\operatorname{span}\{u, v\}$, each point will be expressed by means of its coordinates with respect to the basis $\{u, v\}$. Thus, for instance, $s^{k} \equiv\left(\begin{array}{c}k \\ \frac{1}{k}\end{array}\right)$, for every $k \in \mathbb{N}$.

First, we prove that $C$ is closed. Let $x \in \operatorname{cl} C$, such that $x=\lim _{r \rightarrow \infty} x^{r}$, where $x^{r} \in C$ is also expressed in terms of the basis $\{u, v\}$, for all $r \in \mathbb{N}$. According to Carathéodory's Theorem, there will exist three sequences, in $\mathbb{N}$, $\left\{k_{i}^{r}\right\}_{r=1}^{\infty}, i \in I=\{1,2,3\}$, such that, if $x^{r}=\left(\begin{array}{c}x_{1}^{r} \\ x_{2}^{r}\end{array}\right)$, for every $r \in \mathbb{N}$, we have

$$
\begin{aligned}
& x_{1}^{r} \geq \sum_{i \in I} \lambda_{i}^{r} k_{i}^{r}, \\
& x_{2}^{r}=\sum_{i \in I} \lambda_{i}^{r} \frac{1}{k_{i}^{r}},
\end{aligned}
$$

with $\sum_{i \in I} \lambda_{i}^{r}=1$, and $\lambda_{i}^{r} \geq 0$, for every $i \in I$. 
On the other hand, we can write, w.l.o.g.,

$$
\lim _{r \rightarrow \infty} \lambda_{i}^{r}=\lambda_{i}, i \in I
$$

with $\lambda_{i} \geq 0$, for every $i \in I$ and $\sum_{i \in I} \lambda_{i}=1$.

We consider the set of indices

$$
J:=\left\{i \in I \mid \lambda_{i}>0\right\} .
$$

Taking into account that $\left\{x_{1}^{r}\right\}_{r=1}^{\infty}$ is a convergent (and, consequently, bounded) sequence, it is evident from (2.1) and (2.3) that, for all $i \in J$, the sequence $\left\{k_{i}^{r}\right\}_{r=1}^{\infty}$, is bounded. Thus, for appropiate subsequences, which are denoted in the same way, we can write

$$
\lim _{r \rightarrow \infty} k_{i}^{r}=k_{i}, \quad i \in J
$$

This implies that, from (2.1),

$$
x_{1}=\lim _{r \rightarrow \infty} x_{1}^{r} \geq \sum_{i \in J} \lambda_{i} k_{i}
$$

and, from (2.2),

$$
\begin{aligned}
x_{2} & =\lim _{r \rightarrow \infty} x_{2}^{r}=\lim _{r \rightarrow \infty}\left\{\sum_{i \in J} \lambda_{i}^{r} \frac{1}{k_{i}^{r}}+\sum_{i \notin J} \lambda_{i}^{r} \frac{1}{k_{i}^{r}}\right\} \\
& =\sum_{i \in J} \lambda_{i} \frac{1}{k_{i}}+\limsup _{r \rightarrow \infty} \sum_{i \notin J} \lambda_{i}^{r} \frac{1}{k_{i}^{r}} .
\end{aligned}
$$

Let $\lim \sup _{r \rightarrow \infty} \sum_{i \notin J} \lambda_{i}^{r} \frac{1}{k_{i}^{r}}=\alpha \geq 0$ and take a subsequence such that $\lim _{l \rightarrow \infty} \sum_{i \notin J} \lambda_{i}^{r_{l}} \frac{1}{k_{i}^{r_{l}}}=\alpha$. Since $\frac{1}{k_{i}^{r_{l}}} \leq 1$, for all $l \in \mathbb{N}$, we have

$$
0 \leq \alpha \leq \lim _{l \rightarrow \infty} \sum_{i \notin J} \lambda_{i}^{r_{l}}=0 .
$$

Replacing in (2.4) we obtain

$$
x_{2}=\sum_{i \in J} \lambda_{i} \frac{1}{k_{i}},
$$

hence $x \in C$ and $C$ is closed. According to Corollary 18.3.1 in Rockafellar (1970), every extreme point in $C$ is a point of $S$. In fact, $S$ is the 
set of extreme points in $C$, because the function $h(\alpha)=\frac{1}{\alpha}$ is strictly convex on ] $0,+\infty[$.

Moreover, for every pair $k_{1}, k_{2} \in \mathbb{N} \backslash\{1\}, k_{1} \neq k_{2}$, since $\|u\|=\|v\|=1$, we obtain

$$
\begin{aligned}
\left\|s^{k_{1}}-s^{k_{2}}\right\| & =\left\|\left(k_{1}-k_{2}\right) u-\left(\frac{1}{k_{2}}-\frac{1}{k_{1}}\right) v\right\| \\
& \geq\left|k_{1}-k_{2}\right|-\left|\frac{1}{k_{2}}-\frac{1}{k_{1}}\right| \\
& \geq 1-\frac{1}{2}=\frac{1}{2},
\end{aligned}
$$

and the extreme points of $C$ are isolated, hence $C$ turns out to be quasipolyhedral (apply, for instance, VII, Theorem 1.6 in Bair and Fourneau (1980)).

Nevertheless

$$
\begin{aligned}
A C & =A(\operatorname{conv} S+\operatorname{cone}\{u\})=A(\operatorname{conv} S) \\
& \left.\left.=\operatorname{conv}\left\{\frac{1}{k} A v, k \in \mathbb{N}\right\}=\right] 0,1\right] A v,
\end{aligned}
$$

and this set is not even closed $\left(A v \neq 0_{m}\right)$.

Now, let $D$ be a quasipolyhedral convex set in $\mathbb{R}^{m}$, and let $\hat{P}$ be a polytope in $\mathbb{R}^{n}$, such that $\left(A^{-1} D\right) \cap \hat{P} \neq \emptyset$. Again, we shall prove that it is a polyhedral convex set in $\mathbb{R}^{n}$.

By Theorem 19.3 in Rockafellar (1970), $A \hat{P}$ is a polyhedral convex set in $\mathbb{R}^{m}$, and it is also compact. Then $D \cap(A \hat{P})$ is a polytope in $\mathbb{R}^{m}$, and applying Theorem 19.3 in Rockafellar (1970), $A^{-1}(D \cap(A \hat{P}))$ is a polyhedral convex set in $\mathbb{R}^{n}$. The hypothesis ker $A=\left\{0_{n}\right\}$ allows us to conclude that $A^{-1}(D \cap(A \hat{P}))=\left(A^{-1} D\right) \cap \hat{P}$.

If $C$ is a quasipolyhedral convex set in $\mathbb{R}^{n}$, it is shown in Theorem 5.6 (ii) in Goberna and López (1998) that its set of extreme points has no accumulation point. When a linear operator $A$ is applied, and ker $A \supsetneqq\left\{0_{n}\right\}$, it can happen that a convergent sequence of extreme points appears in $A C$.

Example 2.1 Consider the set, in $\mathbb{R}^{3}, S:=\left\{\left(\begin{array}{c}1 / k \\ 1 / k^{2} \\ k\end{array}\right), k \in \mathbb{N}\right\}$ and the vector $u:=\left(\begin{array}{l}0 \\ 0 \\ 1\end{array}\right)$. We define $C:=\operatorname{conv} S+\operatorname{cone}\{u\} . C$ is a closed convex set whose set of extreme points is $S$, by means of a similar argument for the 
converse statement of Proposition 2.1.

Next we shall prove that $C$ is a quasipolyhedral convex set in $\mathbb{R}^{3}$. Although VII, Theorem 1.6 in Bair and Fourneau (1980) can be again applied, for illustrative purposes, we give an alternative proof which requires the study of the faces of $C$. We denote by

$$
s^{k}:=\left(\begin{array}{c}
1 / k \\
1 / k^{2} \\
k
\end{array}\right), \text { for every } k \in \mathbb{N} .
$$

By Theorem 8.3 in Rockafellar (1970), if $C^{\prime}$ is a non-empty face of $C$, and $S^{\prime}$ is the set of points in $S$ which belong to $C^{\prime}$, then $C^{\prime}=\operatorname{conv} S^{\prime}+$ cone $\{u\}$, in the case $u \in 0^{+}\left(C^{\prime}\right)$, and $C^{\prime}=$ conv $S^{\prime}$ in other case. Moreover, if $C^{\prime}$ is a face other than $C$ itself, then $\operatorname{dim} C^{\prime}<\operatorname{dim} C$ (Corollary 18.1.3 in Rockafellar (1970)).

Now we prove that each four different points in $S$ are affinely independent.

We know that a set of points $\left\{a^{1}, a^{2}, \ldots, a^{m}\right\}$ in $R^{n}$ is affinely independent if and only if the vectors $\left(\begin{array}{c}a^{1} \\ 1\end{array}\right),\left(\begin{array}{c}a^{2} \\ 1\end{array}\right), \ldots,\left(\begin{array}{c}a^{m} \\ 1\end{array}\right)$ are linearly independent in $\mathbb{R}^{n+1}$. Hence, for $k_{1}, k_{2}, k_{3}, k_{4} \in N, k_{1}<k_{2}<k_{3}<k_{4}$,we shall prove that $\left(\begin{array}{c}s^{k_{1}} \\ 1\end{array}\right)$, $\left(\begin{array}{c}s^{k_{2}} \\ 1\end{array}\right),\left(\begin{array}{c}s^{k_{3}} \\ 1\end{array}\right)$ and $\left(\begin{array}{c}s^{k_{4}} \\ 1\end{array}\right)$ are linearly independent in $\mathbb{R}^{4}$.

A direct computation shows that

$$
\operatorname{det}\left(\begin{array}{cccc}
s^{k_{1}} & s^{k_{2}} & s^{k_{3}} & s^{k_{4}} \\
1 & 1 & 1 & 1
\end{array}\right)=\frac{1}{\left(k_{1} k_{2} k_{3} k_{4}\right)^{2}} V\left(k_{1}, k_{2}, k_{3}, k_{4}\right),
$$

where $V\left(k_{1}, k_{2}, k_{3}, k_{4}\right)$ is the determinant of the Vandermonde matrix associated with $k_{1}, k_{2}, k_{3}, k_{4}$. We obtain

$$
\operatorname{det}\left(\begin{array}{cccc}
s^{k_{1}} & s^{k_{2}} & s^{k_{3}} & s^{k_{4}} \\
1 & 1 & 1 & 1
\end{array}\right)=\frac{1}{\left(k_{1} k_{2} k_{3} k_{4}\right)^{2}} \prod_{1 \leq j<i \leq 4}\left(k_{i}-k_{j}\right) \neq 0 .
$$

This also proves that $\operatorname{dim} C=3$.

Therefore, if $C^{\prime}$ is a proper face of $C$, we have the following possibilities:

(1) $C^{\prime}=\operatorname{conv}\left\{s^{k_{j}}, j \in J\right\}$, if $u \notin 0^{+}\left(C^{\prime}\right)$;

(2) $C^{\prime}=\operatorname{conv}\left\{s^{k_{j}}, j \in J-1\right\}+$ cone $\{u\}$, if $u \in 0^{+}\left(C^{\prime}\right)$,

where $J=\left\{1, \ldots, \operatorname{dim} C^{\prime}+1\right\}$. Let us observe that, in case (2), $\operatorname{dim} C^{\prime} \geq 1$ and $\left\{u, s^{k_{1}}, s^{k_{2}}\right\}$ are affinely independent, for every $k_{1}, k_{2} \in \mathbb{N}, k_{1} \neq k_{2}$. 
Hence, all the faces in $C$ are polyhedral (for case (2), we have applied Corollary 19.3.2 in Rockafellar (1970)). This fact allows us to show that, if $P$ is a polytope such that $C \cap P \neq \emptyset$, this intersection set is a polytope too.

If we denote by $\left\{e_{1}, e_{2}, e_{3}\right\}$ the canonical basis of $\mathbb{R}^{3}$, for every pair $k_{1}, k_{2} \in \mathbb{N} \backslash\{1\}, k_{1} \neq k_{2}$,

$$
\begin{aligned}
\left\|s^{k_{1}}-s^{k_{2}}\right\| & =\left\|\left(k_{1}-k_{2}\right) e_{3}-\left(\frac{1}{k_{2}}-\frac{1}{k_{1}}\right) e_{1}-\left(\frac{1}{k_{2}^{2}}-\frac{1}{k_{1}^{2}}\right) e_{2}\right\| \\
& \geq\left|k_{1}-k_{2}\right|-\left|\frac{1}{k_{2}}-\frac{1}{k_{1}}\right|-\left|\frac{1}{k_{2}^{2}}-\frac{1}{k_{1}^{2}}\right| \\
& \geq 1-\frac{1}{2}-\frac{1}{4}=\frac{1}{4},
\end{aligned}
$$

and the extreme points of $C$ are isolated. Hence $P$ only meets a finite number of faces in $C$, and the non-empty intersection of $P$ with each face is a polytope. Consequently, $P \cap \operatorname{bd} C$ is a finite union of polytopes and hence $C \cap P$ is a polytope.

We conclude that $C$ is a quasipolyhedral convex set, and $S$ is its set of (isolated) extreme points.

Consider now the projection of $C$ onto the first two coordinates. Its image set is

$$
\tilde{C}:=\operatorname{conv}\left\{\left(\begin{array}{c}
1 / k \\
1 / k^{2}
\end{array}\right), k \in \mathbb{N}\right\},
$$

which, apart from the fact that it is not closed $\left(0_{2} \in \operatorname{cl} \tilde{C} \backslash \tilde{C}\right)$, has a a convergent sequence of extreme points (the image set of $S$ ).

Corollary 2.1 Let $A$ be a linear transformation from $\mathbb{R}^{n}$ to $\mathbb{R}^{m}$, where $m \geq n$ and $\operatorname{ker} A=\left\{0_{n}\right\}$. If $f$ is a quasipolyhedral convex function on $\mathbb{R}^{n}$ (respectively, $g$ on $\mathbb{R}^{m}$ ), then the convex function Af is quasipolyhedral on $\mathbb{R}^{m}$ (respectively, $g A$ on $\left.\mathbb{R}^{n}\right)$. Moreover, if $g \in \mathcal{Q}^{m}$, then $g A \in \mathcal{Q}^{n}$.

Proof. For every $y \in \mathbb{R}^{m}$,

$$
\begin{aligned}
(A f)(y) & =\inf \{f(x) \mid A x=y\} \\
& =\inf \left\{f(x) \mid x \in A^{-1}\{y\}\right\} .
\end{aligned}
$$

Since ker $A=\left\{0_{n}\right\}$, the set $A^{-1}\{y\}$ is either a singleton or empty. This fact leads us to conclude that, even in the case where $f$ is finite-valued, $A f$ will be $+\infty$ in those points $y$ such that $A^{-1}\{y\}=\emptyset$. Otherwise, $(A f)(y)<+\infty$, in which case we can write

$$
(A f)(y)=f\left(A^{-1}\{y\}\right) .
$$


Consider now the linear transformation, from $\mathbb{R}^{n+1}$ to $\mathbb{R}^{m+1}$,

$$
\bar{A}:\left(\begin{array}{c}
x \\
x_{n+1}
\end{array}\right) \rightarrow\left(\begin{array}{c}
A x \\
x_{n+1}
\end{array}\right)
$$

where $x \in \mathbb{R}^{n}$ and $x_{n+1} \in \mathbb{R}$. Clearly, $\operatorname{ker} \bar{A}=\left\{0_{n+1}\right\}$, and we have

$$
\begin{aligned}
\bar{A}(\text { epi } f) & =\left\{\left(\begin{array}{c}
A x \\
x_{m+1}
\end{array}\right) \in \mathbb{R}^{m+1} \mid f(x) \leq x_{m+1}\right\} \\
& =\left\{\left(\begin{array}{c}
y \\
x_{m+1}
\end{array}\right) \in \mathbb{R}^{m+1} \mid f\left(A^{-1}\{y\}\right) \leq x_{m+1}\right\} \\
& =\operatorname{epi}(A f) .
\end{aligned}
$$

Applying Proposition 2.1, epi $(A f)$ is a quasipolyhedral convex set in $\mathbb{R}^{m+1}$, hence $A f$ is quasipolyhedral.

On the other hand, since

$$
(g A)(x)=g(A x),
$$

for every $x \in \mathbb{R}^{n}$, and taking into account that

$$
(\bar{A})^{-1}(\text { epi } g)=\left\{\left(\begin{array}{c}
x \\
x_{n+1}
\end{array}\right) \in \mathbb{R}^{n+1} \mid g(A x) \leq x_{n+1}\right\}=\operatorname{epi}(g A),
$$

it follows that $g A$ is quasipolyhedral and, in particular, if $g$ is finite-valued, $(g A)(x)<+\infty$, for every $x \in \mathbb{R}^{n}$.

Corollary 2.2 Let $A$ be a non-singular linear transformation from $\mathbb{R}^{n}$ to $\mathbb{R}^{n}$. If $f \in \mathcal{Q}^{n}$, then $A f \in \mathcal{Q}^{n}$.

Proof. This is immediate from Corollary 2.1 and the fact that $A^{-1}\{y\} \neq \emptyset$, for every $y \in \mathbb{R}^{m}$.

Proposition 2.2 If $f_{1}, f_{2} \in \mathcal{Q}^{n}$, then $f_{1}+f_{2} \in \mathcal{Q}^{n}$.

Proof. According to Proposition 1.1, consider the representations of $f_{1}$ and $f_{2}$,

$$
\begin{aligned}
& \sigma_{1}=\left\{\left\langle a_{t}, x\right\rangle-x_{n+1} \leq b_{t}, t \in T\right\}, \\
& \sigma_{2}=\left\{\left\langle c_{s}, x\right\rangle-x_{n+1} \leq d_{s}, s \in S\right\},
\end{aligned}
$$

respectively, verifying that, for every non-empty compact set $C$ in $\mathbb{R}^{n}, T(C)$ and $S(C)$ are non-empty and finite sets. 
For each $(t, s) \in T \times S$, we define

$$
\begin{aligned}
& e_{t, s}:=a_{t}+c_{s}, \\
& h_{t, s}:=b_{t}+d_{s} .
\end{aligned}
$$

Consider the system

$$
\sigma:=\left\{\left\langle e_{t, s}, x\right\rangle-x_{n+1} \leq h_{t, s},(t, s) \in T \times S\right\} .
$$

We shall prove that $\sigma$ is a representation of $f_{1}+f_{2}$; i.e.,

$$
\left(f_{1}+f_{2}\right)(x)=\sup \left\{\left\langle e_{t, s}, x\right\rangle-h_{t, s},(t, s) \in T \times S\right\},
$$

for all $x \in \mathbb{R}^{n}$.

Since $f_{1}(x) \geq\left\langle a_{t}, x\right\rangle-b_{t}$, for all $t \in T$, and $f_{2}(x) \geq\left\langle c_{s}, x\right\rangle-d_{s}$, for all $s \in S$, it follows that

$$
\left(f_{1}+f_{2}\right)(x) \geq\left\langle e_{t, s}, x\right\rangle-h_{t, s}, \text { for all }(t, s) \in T \times S .
$$

On the other hand, $T(x) \neq \emptyset$ and $S(x) \neq \emptyset$, hence $(t, s) \in T(x) \times S(x) \subset$ $T \times S$ satisfies

$$
\left(f_{1}+f_{2}\right)(x)=\left\langle e_{t, s}, x\right\rangle-h_{t, s} .
$$

Now, we shall show that this representation of $f_{1}+f_{2}$ verifies that, for every non-empty compact set $C$ in $\mathbb{R}^{n},(T \times S)(C)$ is non-empty and finite. Then, by Proposition 1.1, it follows that $f_{1}+f_{2} \in \mathcal{Q}^{n}$.

We already know that, for all $x \in C, \emptyset \neq T(x) \times S(x) \subset(T \times S)(x)$. Then $(T \times S)(C) \neq \emptyset$.

On the other hand, $T(C)$ and $S(C)$ are finite sets. We shall prove that $(T \times S)(C) \subset T(C) \times S(C)$. Take $(t, s) \in(T \times S)(C)$. Then, for some $x \in C$,

$$
\left(f_{1}+f_{2}\right)(x)=\left\langle e_{t, s}, x\right\rangle-h_{t, s} .
$$

But $f_{1}(x) \geq\left\langle a_{t}, x\right\rangle-b_{t}$ and $f_{2}(x) \geq\left\langle c_{s}, x\right\rangle-d_{s}$, and none of them can be a strict inequality. Hence, $t \in T(C)$ and $s \in S(C)$. It follows that $(T \times S)(C) \subset T(C) \times S(C)$, and $(T \times S)(C)$ is finite.

Proposition 2.3 Let $C$ be a quasipolyhedral convex set in $\mathbb{R}^{n}$. Then $\lambda C$ is a quasipolyhedral convex set in $\mathbb{R}^{n}$, for every $\lambda \in \mathbb{R}$.

Proof. We consider the linear transformation $A$ from $\mathbb{R}^{n}$ to $\mathbb{R}^{n}, x \rightarrow \lambda x$. Then either $A x=0_{n}$, for all $x \in \mathbb{R}^{n}$, (when $\lambda=0$ ) or ker $A=\left\{0_{n}\right\}$, and applying Proposition 2.1, $\lambda C=A C$ is a quasipolyhedral convex set in $\mathbb{R}^{n}$.

Corollary 2.3 If $f \in \mathcal{Q}^{n}$, then $f \lambda \in \mathcal{Q}^{n}$, for every $\lambda>0$. 
Proof. First, it is evident that $f \lambda$ is finite-valued, since $(f \lambda)(x):=$ $\lambda f\left(\lambda^{-1} x\right)<+\infty$, for every $x \in \mathbb{R}^{n}$.

On the other hand, we have

$$
\begin{aligned}
\operatorname{epi}(f \lambda) & =\left\{\left(\begin{array}{c}
x \\
x_{n+1}
\end{array}\right) \in \mathbb{R}^{n+1} \mid \lambda f\left(\lambda^{-1} x\right) \leq x_{n+1}\right\} \\
& =\left\{\left(\begin{array}{c}
\lambda y \\
\lambda y_{n+1}
\end{array}\right) \in \mathbb{R}^{n+1} \mid f(y) \leq y_{n+1}\right\} \\
& =\lambda(\operatorname{epi} f)
\end{aligned}
$$

It follows that $f \lambda$ is a quasipolyhedral function, according to Proposition 2.3.

Remark 2.1 In the case $\lambda=0$,

$$
(f 0)(x)=\delta\left(x \mid 0_{n}\right)=\left\{\begin{array}{cc}
0, & \text { if } x=0_{n}, \\
+\infty, & \text { if } x \neq 0_{n}
\end{array},\right.
$$

for any proper convex function $f$ on $\mathbb{R}^{n}$. Hence, epi $(f 0)=\left\{0_{n}\right\} \times \mathbb{R}^{+}$, which is a quasipolyhedral convex set (in fact, it is polyhedral) and $f 0$ is a quasipolyhedral convex function with dom $(f 0)=\left\{0_{n}\right\}$.

Finally, what can be said about the recession function of a finitevalued quasipolyhedral function $f$ ? First, we shall see that the concept of quasipolyhedral convex function has no meaning for $f 0^{+}$.

Proposition 2.4 Let $K$ be a convex cone in $\mathbb{R}^{n}$. Then $K$ is quasipolyhedral if and only if it is polyhedral.

Proof. Applying VII, Theorem 1.6 in Bair and Fourneau (1980), a non-empty convex set $C$ is quasipolyhedral if and only if the cone of feasible directions at $x \in C$,

$$
D_{C}(x):=\left\{y \in \mathbb{R}^{n} \mid x+\alpha y \in C, \text { for some } \alpha>0\right\},
$$

is polyhedral, for every $x \in C$.

In particular, if $K$ is a cone, $0_{n} \in K$, and

$$
D_{K}\left(0_{n}\right)=\left\{y \in \mathbb{R}^{n} \mid \alpha y \in K, \text { for all } \alpha \geq 0\right\}=K .
$$

It follows that, if $K$ is quasipolyhedral, then $D_{K}\left(0_{n}\right)=K$ is polyhedral, and the converse statement is always true.

Since epi $\left(f 0^{+}\right)=0^{+}$(epi $f$ ), according to this result, the question that arises is if $f 0^{+}$will be a polyhedral convex function when $f$ is a finite-valued 
quasipolyhedral function. But, if $C$ is a quasipolyhedral convex set, $0^{+} C$ is not a polyhedral convex cone, in general. Example 5.1 in Goberna and López (1998) provides a quasipolyhedral convex set whose recession cone is not polyhedral (Exercise 9.8 in Goberna and López (1998)).

\section{References}

Bair, J. and Fourneau, R. (1980). Etude Géometrique des Espaces Vectoriels II: Polyédres et Polytopes Convexes. Springer-Verlag, Berlin.

Fajardo, M.D., López, M.A. and Puente, R. (2006). (Locally) Farkas representations and quasipolyhedrality of a convex function. Alicante University, manuscript.

Goberna, M.A., and López, M.A. (1998). Linear Semi-Infinite Optimization. John Wiley and Sons, Chichester, U.K.

Rockafellar, R.T. (1970). Convex Analysis. Princeton University Press, Princeton, New Jersey. 